Please send trade news information and illustrations to Arveen Bajaj at the $B D J$, Nature Publishing Group, The Macmillan Building, 4-6 Crinan Street, London N1 9XW.

Trade news is provided as a service to readers using text and images from the manufacturer, supplier or distributor and does not imply endorsement by the $B D J$. Normal and prudent research should be exercised before purchase or use of any product mentioned.

\section{New to range}

Henry Schein has announced the addition of Kavo's HealOzone to its portfolio.

The HealOzone has been specially enhanced for use in root canal procedures, enabling pain-free treatment which can be completed in a single session. By eliminating the need for drilling, the HealOzone is particularly suitable for children and phobic patients in the treatment of root canals and pit and fissure caries. Other possible treatments could include orthodontic problems, cervical sensitivity and soft tissue problems.

The HealOzone is an easy to use, with neither dentist, nurse nor patient exposed to ozone.

It destroys 99.9\% of caries bacteria in 20 seconds with the maximum retention of healthy tooth substance, considerably reducing the need for fillings.

Reader response number 51

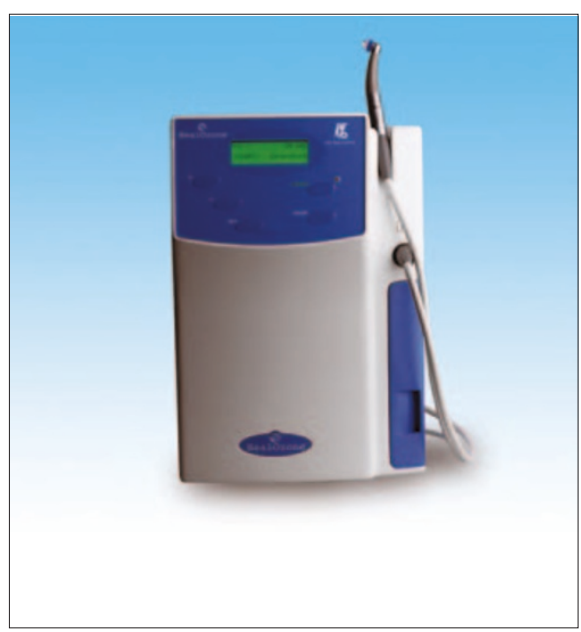

\title{
Quick pain relief
}

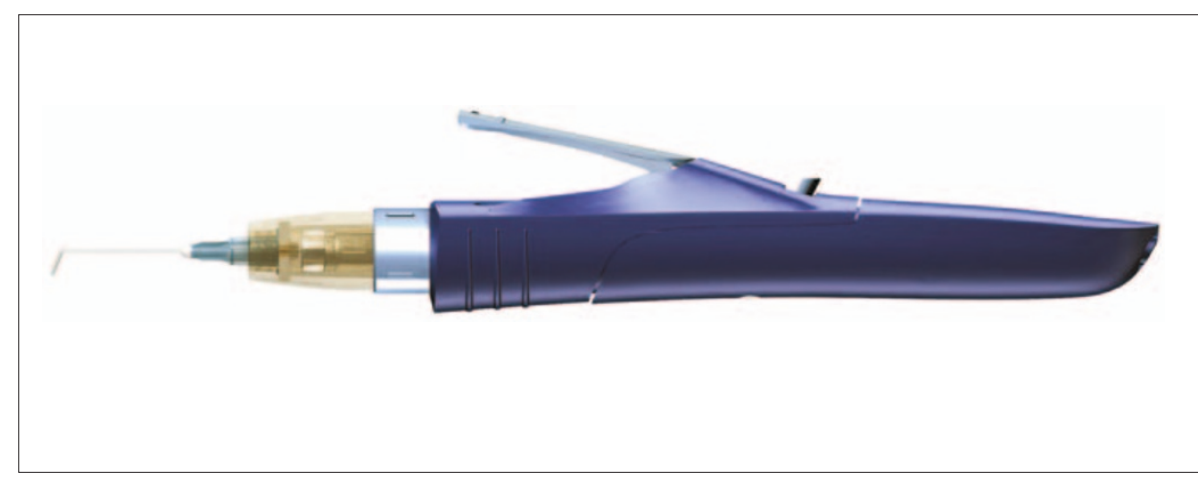

Oraqix periodontal gel (lidocaine, prilocaine) from Dentsply Ltd is a non-injectable dental local anaesthetic. It is indicated in adults for localised anaesthesia in periodontal pockets for diagnostic and treatment procedures such as probing, scaling and/or root planing (SRP).

There is no need for a needle for those who are phobic - and it is a quick option

\section{Durability and strength}

Unotrays from The Dental Directory are constructed from durable plastic for optimum strength but are pre-formatted to ensure the impression material is retained. Each box contains 25 trays of one size and one handle. UnoDent Imprep impression material offers dentists a variety of choices in terms of consistency and setting times. The material has low shrinkage properties and high thixotrophy and is available in both condensation cured and addition cured kits and cartridges.

Imprep is non-toxic and biocompatible in accordance with European regulations. Both putties and pastes are suitable for different applications: wash technique, double mixing etc and are available in a range of colours to enable the user to customise impressions.

Reader response number 52 to provide pain relief during SRP for those who normally have to go without. Oraqix is applied to the periodontal pocket with a special patient-friendly, ergonomic Oraqix Dispenser, developed by Dentsply. The anaesthetic takes effect in around 30 seconds.

Reader response number 50

\section{Infection control solution}

The new disposable dental kit from Henry Schein represents revolution in health and safety standards for dentists treating patients who present a high risk of infection. The kit comprises a tray with a range of disposable instruments, including a stainless steel tipped probe, tweezers and a mirror, along with a patient bib, napkin, two cotton rolls and two ear loop masks. All the items are biodegradable except the mirror, which can be given to the patient as a souvenir. Using the kit naturally eliminates the need for sterilising instruments after use, which has substantial cost and time-saving benefits. The small, sterile package is stackable for convenient storage and is sold in boxes with a minimum of 50 kits per box.

Reader response number 53 

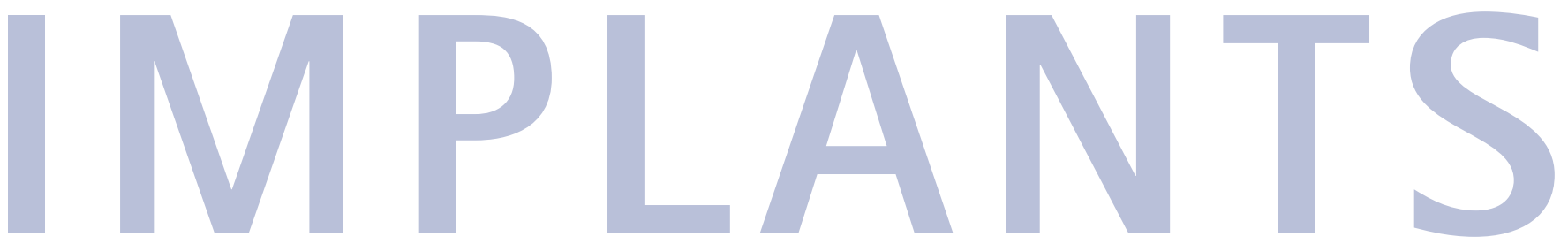

\section{New internal hex implant system}

The Imtec Corporation has recently introduced Endure, an internal hex implant system. The simplicity of the Endure system allows it to be placed as a one or a two-stage implant and it can be used for crowns, bridges and dentures. It features variable implant positioning for natural, complementary dentition and all prosthetic components are universally compatible with all lengths and both

diameter sizes. The
product is available
in 3.5 and $4.3 \mathrm{~mm}$
diameters and
lengths of 11, 14 and
17mm. For more
information contact
www.imtec.com.
Reader response
number $\mathbf{5 4}$

\section{Tailored to implant patients}

The Implant kit from Molar contains a comprehensive range of products, specifically for use by the implant patient. The kit includes a Special Care toothbrush and a TePe Select Extra soft toothbrush for general oral hygiene, and for specific cleaning around implant abutment posts, the Implant and Interspace brush can be used. Sample packs of the TePe Interdental brushes and Proximal brush are included, as is a toothbrush travel case which can be used for any of the TePe toothbrushes. Also included are Plastic interdental sticks and Proxi-Floss.

The Implant
kit is available
from Molar
for £9.90 each
with a minimum
order quantity
of three packs.
Reader response
number 56

\section{New alginate syringe launched}

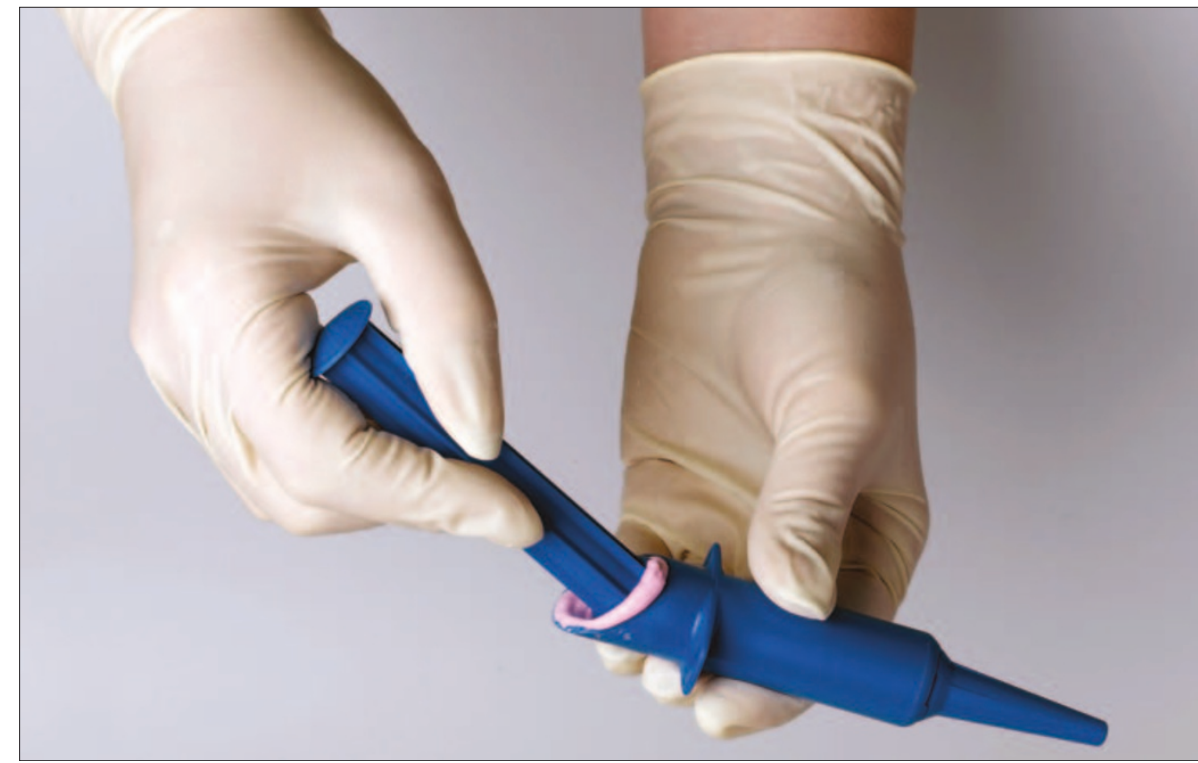

The new Alma alginate syringe from Astek Innovations enables dentists to precisely place alginate into difficult to reach areas of the mouth and means that finger application of potentially messy alginate impression material becomes a thing of the past. to carry just enough alginate into the
The Alma syringe is specially designed tuberosity and sulcus areas. It can be loaded in a few seconds from the main alginate mix and is applied whilst the tray is being filled so there is no loss in time. It is easily cleaned post-op and can then be autoclaved. A special introductory offer for a limited period reduces the price from $£ 12.95$ to $€ 9.95$ (excluding vat).

Reader response number 55

\section{Handpieces for implant surgery}

The Sirona T1 Classic handpieces are manufactured from high-grade titanium to provide a smooth, robust and extremely balanced instrument ideally designed for implant surgery. The fibre optic light guide provides optimum illumination with 25,000 lux output for visualising every area within the operating site.

Together with the SiroTorque L electric motor, the Sirona Classic 24:1 implant handpiece allows you to provide all necessary treatment for implantology with just one handpiece. Coolant is delivered directly to the operating site with the Kirschner/Meyer principle for optimum treatment with irrigation.

Noise levels for the Classic implant handpieces have been reduced giving less stress to user and patient whilst treatment is carried out. The smooth titanium surface is free of all potential dirt traps making the handpiece easy to repeatedly clean and sterilise in an autoclave at up to $135^{\circ} \mathrm{C}$.

Reader response number 57

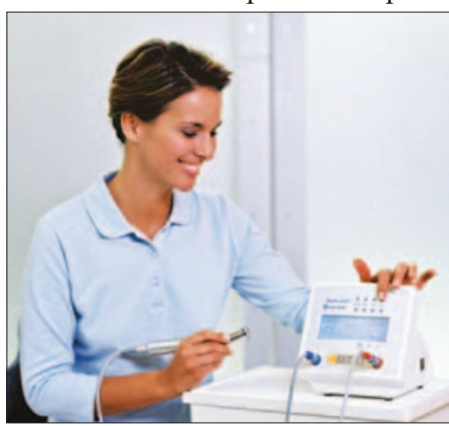




\section{Sterile silicone launched}

Zhermack have launched a unique sterile silicone for implant techniques which require impressions immediately after surgery. Elite Implant is sterile to reduce the risk of post-operative complications and is biocompatible and radiopaque to avoid tissue damage if a fragment remains in the mucosa. As well as the benefits Elite Implant offers peace of mind over the most commonly used materials for implants which studies show can be cytotoxic if a fragment remains.

Elite Implant fits standard dispensers and comes in three viscosities. "Heavy(50ml)" is used on its own for edentureless patients and is extra rigid. "Medium(50ml)" is used on its own for partially edentate patients or with "Light $(5 \mathrm{ml})$ " which is used around the implants and preparations.

Reader response number 58

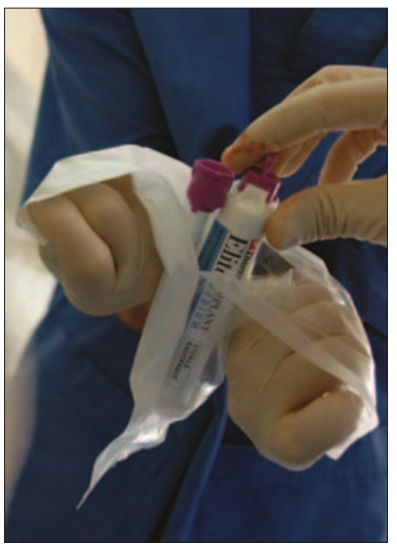

\section{Innovative implant designs}

3i Implant Innovations has introduced two new implant products to its range. Firstly the Certain Prevail implant features integrated platform switching, which eliminates any mismatching of components. This provides a simple and predictable option for the potential to achieve enhanced crestal bone preservation, which may result in more predictable soft tissue aesthetics. The implant incorporates a coronal bevel design that medialises the implant-to-abutment junction, potentially enhancing crestal bone preservation. The appropriately colour coded prosthetic table ensures a correct match with existing Certain Abutments and its entire length is treated with the osseotite surface with expanded collar for maximum stability. In addition, the Certain QuickSeat Connection provides the clinician with an audible and tactile click that confirms the abutment is properly seated.

Secondly the Provide Restorative System provides easily identifiable colourcoded compatible parts, with two post heights ( 4 and $5.5 \mathrm{~mm}$ ). It features the Certain QuickSeat Connection, and its snapon impression components eliminate the need for a fixture-level impression. For the

\section{Hydrophilic surface technology}

The Straumann implant range now includes its new active and hydrophilic surface technology, SLActive. The SLActive surface optimises implant stability in the critical early treatment period, thereby enhancing treatment. It provides 60\% more bone after two weeks, reduces healing time to as little as 3-4 weeks and enables practitioners to offer new options by maximising treatment predictability.

Reader response

number 60

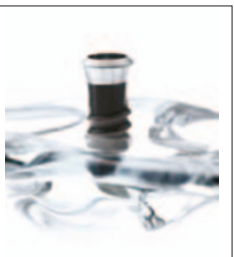

surgeon, Provide Abutments offer the ability to choose between four collar height options (1, 2, 3 and $4 \mathrm{~mm}$ ). This gives the surgeon implant placement flexibility as the collar can be optimally selected when the implant is ready to restore, rather than when the implant is placed. Additionally, because the implant is placed at bone level and not transgingivally, the final crown margin is not pre-determined at the time of surgery. In the event of soft tissue changes, the Provide Abutment allows the surgeon the option of changing the abutment at a later date.

Reader response number 59

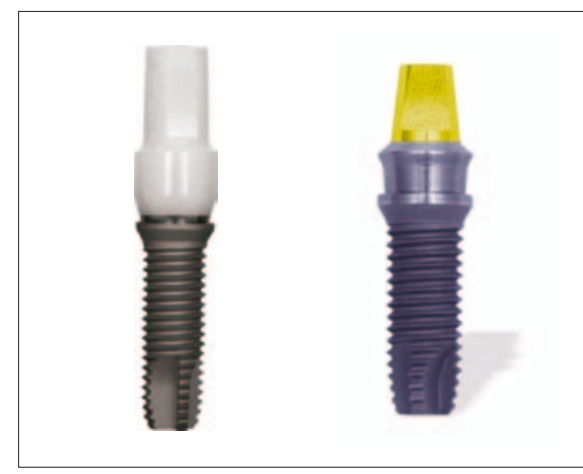

\section{Synthetic regeneration}

SynthoGraft is a synthetic, biocompatible and resorbable granulate ceramic made of pure-phase beta-tricalcium phosphate $\left(\mathrm{Ca}_{3}\left(\mathrm{PO}_{4}\right)_{2}\right)$ which is used as a matrix for oral and maxillofacial bone regeneration or augmentation. SynthoGraft's unique structure provides increased stability and its micro-porosity and nano-porosity allow for rapid vascularisation and subsequent resorption. It also achieves the greatest efficacy when mixed with the patient's own blood. Other additives, including autogenous bone particles, are not needed or recommended.

Reader response number $\mathbf{6 1}$ 